\title{
Zur Relevanz von sozialer Ungleichheit im Kontext der Mediensozialisationsforschung
}

Ingrid Paus-Hasebrink

\begin{abstract}
Ziel des vorliegenden Beitrags ist es, deutlich zu machen, dass Mediensozialisationsforschung nicht ohne den Blick auf die Lebenswelt Heranwachsender auskommen kann; diese wird geprägt vom sozialen Milieu (einer Manifestation des je spezifischen Zusammenhangs von sozialer Lage und Lebensentwurf der agierenden Personen), in dessen Rahmen sich das Aufwachsen von Kindern und Jugendlichen vollzieht. Seine spezifischen Konstellationen, zu denen auch die Medienumgangsweisen gehören, müssen daher in die Forschung miteinbezogen werden. Um die Bedeutung, die Mediensymbolik im Alltag von Heranwachsenden einnimmt, im Kontext untersuchen zu können, erscheint eine integrative sowie interdisziplinäre Forschung notwendig. Als Beispiel dafür wird eine Panelstudie zur (Medien-)Sozialisation bei sozial benachteiligten Kindern in Österreich in ihrer theoretischen und methodologischen Anlage vorgestellt.
\end{abstract}

\section{Sozialisationsforschung als Mediensozialisationsforschung}

Das pädagogische und sozialisatorische Ziel gelingenden Aufwachsens von Kindern und Jugendlichen kann nicht ohne Blick auf ihre Medienwelt(en) erreicht werden (vgl. Fromme 2007, S. 18). Obwohl sich Kinder - wie auch Jugendliche - jeweils unterschiedlicher Medien und Medienangebote bedienen, um die für ihr jeweiliges Alter relevanten und dominanten Entwicklungsaufgaben zu lösen und die damit verbundenen Krisen zu bewältigen (vgl. Vollbrecht 2007, S. 102), spielen Medien und kindliche Umgangsweisen mit ihnen in den allgemeinen Sozialisationstheorien bislang kaum eine Rolle; die Beschäftigung damit lässt sich bestenfalls als ein Randphänomen im Kontext der Sozialisationsforschung bezeichnen (vgl. Hoffmann 2007). Die hohe Bedeutung, die Mediensymbolik im Alltag von Heranwachsenden einnimmt, verlangt somit nach einer adäquaten Beachtung von Mediengeboten und Rezeptionsweisen im Kontext der Erforschung von Sozialisationsprozessen; denn Mediensymbolik spielt im Prozess der Entwicklung und Sozialisation von Kindern und Jugendlichen für die Sach-, Selbst- und Sozialauseinandersetzung auf allen Ebenen des Alltags eine zentrale Rolle. Ebenso wichtig ist es aber mitzubedenken, dass mediale Angebote nie direkt auf Kinder und Jugendliche einwirken (vgl. Spanhel 2006, S. 54); entscheidend ist vielmehr der soziale Rahmen in ihren Familien, in Kindergarten, Hort, Heim oder Schule leben - es handelt sich immer 
um rekursive Prozesse - sodass die sozialen Hintergründe, in denen Heranwachsende leben, in die Forschung miteinbezogen werden müssen.

Wenn von Medien die Rede ist - dies kommt noch hinzu -, dann zumeist nur aus einer disziplinären Perspektive, entweder aus der soziologischen, psychologischen, aus der kommunikations- oder aus der erziehungswissenschaftlichen (vgl. Sutter 2007, S. 135). Diese Herangehensweise greift aber zu kurz, da sich das Medienhandeln von Kindern und Jugendlichen im jeweiligen Alltag mit seinem je spezifischen Bedingungsgeflecht sozialer und individueller Faktoren abspielt (vgl. Paus-Haase 1999). Mediensozialisationsforschung bedarf einer interdisziplinären Herangehensweise (vgl. Geulen 2004, S. 19, Sutter 2004, S. 94), um der Komplexität und Vielfalt des Medienhandelns junger Menschen im lebensweltlichen Zusammenhang gerecht zu werden.

Mediensozialisationsprozesse zu erforschen bedeutet, der Rolle von Medien im Gesamtkontext von Sozialisationsprozessen mit Hilfe eines theoretisch und methodologisch (mithin auch methodisch) aufeinander abgestimmten, kohärenten Vorgehens in ihrer Komplexität und Vielfalt zu erfassen und "passiv-monokausale Erklärungsmuster» (Wegener 2007, S. 460) zugunsten einer individuelle, soziale, kulturelle und sozialisatorischer Effekte mitreflektierenden Medienforschung zu vermeiden (vgl. ebd).

\section{Soziale Armut und Kinder(medien)-Alltag}

Soziale Lebensbedingungen von Menschen finden, so lässt sich feststellen, ihren Ausdruck im Kinder- und Jugendalltag. Sie formieren unterschiedliche Modi des Aufwachsens und damit insgesamt von Kindheit und Jugend und setzen je spezifische Sozialisationsbedingungen; denn die materielle, wirtschaftliche, soziale und mentale Umwelt findet ihren Niederschlag in den Erlebnisräumen von Kindern, nimmt Einfluss auf die Erziehungsstile ihrer Eltern, bestimmt «Kargheit oder Wohlhabenheit» (Hugger 1998, S. 33). Soziale Benachteiligung wirkt sich deutlich auf die Sozialisation von Kindern und Jugendlichen aus; so wurde in unterschiedlichen Forschungsstudien der enge Zusammenhang zwischen ökonomisch benachteiligten Lebenslagen von Familien und dem Bildungsniveau der Eltern hervorgehoben; und da eine notwendige Balancierung von selbst- und fremdbestimmten Handlungsperspektiven in individuierten, modernen Gesellschaften sowieso besonders erschwert ist - dies gilt für Familien ebenso wie für die darin aufwachsenden Kinder; Heranwachsende sind bei der Suche nach sich selbst häufig auf sich allein gestellt (vgl. Grundmann 2000, S. 95f.) - gebührt sozial benachteiligten Kindern und Jugendlichen in der Mediensozialisationsforschung besondere Beachtung. Sie haben es, wie auch andere Heranwachsende, mit einer Fülle von nicht einfach zu bewältigenden Aufgaben zu tun, dies jedoch unter erschwerten Bedingungen. Denn vor allem das Milieu, in dem eine Familie lebt, spielt im Sozialisationsprozess eine wichtige Rolle. Es verstärkt «Erfahrungswidersprüche» (vgl. Grundmann 2004, 
S. 327), denen eine hohe Relevanz im Kontext von Sozialisationsprozessen beigemessen werden muss, da diese durch den je spezifischen «Umgang mit Ambivalenzen» (2004, S. 327) geprägt werden, die «aus Spannungen im Sozialgefüge, wie sie etwa durch Altersdifferenzen zwischen Eltern und Kindern, durch Geschlechterdifferenzen in Paarbeziehungen oder durch widersprüchliche Rollenerwartungen und Rollenambiguitäten zustande kommen» (ebd). Schliesslich werden die neben den subjektiven Dimensionen, wie etwa erfahrungsbiographisch bedingten Verschiedenheiten, den Sozialisationsprozess mitprägenden Erfahrungen der sozialen Akteure durch «differente, generative Lagen und sozioökonomische Positionen im sozialen Raum (institutionelle Dimensionen) hervorgerufen» (ebd). Die Chancen für die Ausbildung einer «autonomen Identität», eine zentrale normative Zielvorstellung von Sozialisationsprozessen, sind ungleich verteilt. ${ }^{1}$

Zwar erscheint Kindheit und Jugend heute deutlich homogener als zu früheren Zeiten, denn auch Kinder partizipieren am insgesamt gestiegenen Wohlstand und geniessen einen in der Gesellschaft höheren Level sozialer Sicherheit. Dennoch ist in Österreich zum Beispiel laut Daten von Statistik Austria ein Viertel der Kinder und Jugendlichen unter 20 Jahren (27\%) armutsgefährdet. ${ }^{2}$ Besonders von Armut gefährdet sind Alleinerzieher/innen und deren Kinder, Grossfamilien oder Familien mit ausländischer Abstammung (vgl. Statistik Austria 2007, S. 15). ${ }^{3}$ Vor allem Kinder aus süd-/osteuropäischen Migrantenfamilien leben, so ein Ergebnis für Deutschland, in Armut (vgl. Bundesministerium für Familie, Senioren, Frauen und Jugend 2005). Bei Familien mit drei oder mehr Kindern und bei Familien mit kleineren Kindern, vor allem wenn die Frau nicht erwerbstätig ist, sowie insbesondere bei Ein-Eltern-Familien zeigt sich eine überdurchschnittliche Armutsgefährdung (vgl. Statistik Austria 2007, S. 15). Manifeste Armut - niedriges Einkommen sowie niedriger Lebensstandard - tritt immerhin bei fünf Prozent der Bevölkerung

1 Grundmann hebt hervor, dass nicht sozio-ökonomische und sozio-kulturelle Bedingungen an sich, sondern die Interaktions- und Handlungskompetenz der Eltern die Entwicklungsprozesse und Erfahrungen der Kinder und Jugendlichen mitprägen (vgl. ebd); sie sind als Basis für die Entwicklung von Identität und Selbstbild im Kontext der Sozialisation vor dem Hintergrund der kindlichen Lebenswelt zentral.

2 Armutsgefährdung liegt vor, wenn nach europäischer Konvention in den Hauhalten nur $60 \%$ des Medianäquivalenzeinkommens erzielt werden kann; das sind in Österreich für einen Einpersonenhaushalt 10.796 Euro im Jahr bzw. 900 Euro im Monat. Die Armutsgefährdungsquote wird für Österreich mit 12 Prozent angegeben (vgl. Statistik Austria 2007). «EU-SILC ist eine Erhebung über Einkommen und Lebensbedingungen, in deren Rahmen alle Informationen erhoben werden, die notwendig sind, um ein umfassendes Bild über die Lebenssituation von Menschen in Privathaushalten zu gewinnen. Seit 2004 wird EU-SILC als integrierte Quer- und Längsschnitterhebung durchgeführt, das heisst die teilnehmenden Haushalte werden bis zu vier Jahre zur Lebens- und Einkommenssituation, zu Kinderbetreuung, Gesundheit usw. befragt und leisten damit einen wichtigen Beitrag, die Lebensbedingungen verschiedenster Bevölkerungsgruppen abbilden zu können. Ab 2005 wird EU-SILC in allen EU-Mitgliedstaaten und einigen weiteren Ländern durchgeführt und somit nicht nur in Österreich sondern auch auf Europäischer Ebene die Datengrundlage für den Bereich Einkommen, Armut und soziale Teilhabe bilden» (http://www.statistik.at/web_de/dynamic/services/publikationen/6/publdetail?id=6\&listid=6\&detail=312).

3 Gleiche Bedingungen lassen sich für Deutschland nachweisen (vgl. AWO 2000, S. 5). 
auf; bei sieben Prozent ist Einkommensarmut festzustellen, aber keine mangelnde Teilhabe in zentralen Lebensbereichen. Aber immerhin 21 Prozent der Personen mit einem Einkommen über der Armutsgefährdungsschwelle sind in Österreich in zentralen Lebensbereichen stark eingeschränkt. Sozialleistungen für Niedrigeinkommen können diese Problematik zwar mindern, jedoch nicht ausgleichen (vgl. ebd). ${ }^{4}$ Als Ursachen für den seit der Mitte der 1980er Jahre bestehenden Trend zunehmender Armut nennt Palentien vor allem die Zunahme der Arbeitslosigkeit, veränderte Familien- bzw. Haushaltsformen sowie zurückgehende Sozialleistungen, aber auch die Notwendigkeit einer steigenden Mobilität, Flexibilität und einer stetigen Weiterqualifizierung von Erwerbstätigen (vgl. Palentien 2003, S. 91).

Auf die hohe Relevanz ungleicher gesellschaftlicher Lebensbedingungen ${ }^{5}$ haben bereits Untersuchungen Ende der 1950er Jahre hingewiesen. Beinstein etwa hat den Zusammenhang von schichtspezifischem Sprachgebrauch und Sozialschicht nachgewiesen (vgl. Bernstein 1961) und Kohn einen schichtspezifischen Zusammenhang zum Erziehungsverhalten von Eltern deutlich gemacht (vgl. Kohn 1969). Insbesondere die Studien von Bourdieu ${ }^{6}$ weisen auf die grosse Bedeutung milieubedingter Faktoren hin (vgl. Bourdieu 1982).

In seinen Studien zeigte Wilhelm Heitmeyer starke Tendenzen, sozialer Segmentierung' auf; er gab zu bedenken, dass nicht alle Heranwachsenden gleichermassen über die entsprechenden Handlungskompetenzen zur Bewältigung ihres Alltags verfügen und verwies auf Ungleichheiten beim Zugang zu ,begehrten Lebenschancen'; Heitmeyer formulierte schon 1987 die These, dass nur die Starken fortschreitenden Individualisierungstendenzen gewachsen seien (vgl. Heitmeyer 1987). 1990 warnte Iben, dass sozial benachteiligte Kinder durch ihre Lebenssituation verursacht eine Reihe von Belastungen und Erschwernissen ausgesetzt sind: «Der Rückgang elementarer Sozialerfahrungen, auch durch den in bestimmten Schichten ex-

4 Laut der Armutsdefinition der Europäischen Gemeinschaft aus dem Jahr 1985 spricht man von «verarmten Personen, Einzelpersonen, Familien und Personengruppen» wenn sie «über so geringe (materielle, kulturelle und soziale) Mittel verfügen, dass sie von der Lebensweise ausgeschlossen sind, die in dem Mitgliedstaat, in dem sie leben, als Minimum hinnehmbar ist.»

5 Ein Blick auf die Ergebnisse der sozialen Ungleichheitsforschung macht deutlich, dass es keine linearen «Wenn-dann-Mechanismen» gibt, die von einer speziellen sozialen Ausgangssituation eines Kindes (wie z. B. Schichtzugehörigkeit der Eltern) auf eine bestimmte Konsequenz im Verlauf seiner Sozialisation schliessen lassen (z. B. Lernbehinderung). Ging man bis zu Beginn der 1980er Jahre von Schichten- oder Klassenmodellen aus, die eine Einteilung aufgrund von wenigen Dimensionen vornahmen, so wurden in neueren Modellen andere Begriffe gewählt, um der Vielschichtigkeit und Komplexität an Charakteristika Rechnung zu tragen, die die soziale Situation eines Menschen oder einer Familie im Verhältnis zur Gesellschaft, in der sie lebt, bestimmt (vgl. Burzan 2004, S. 12f.). Neue Bezeichnungen wie «Lebensstil», «Milieu» oder «soziale Lage» kamen auf, mit denen versucht wurde, die unterschiedlichen Lebenskonstellationen der Menschen der Wirklichkeit besser entsprechend zu erfassen und abzubilden. Diese Begriffe sind jedoch nicht synonym zu verstehen, wie Weiss betont (1997); siehe zum Verständnis dieser Begriffe im vorliegenden Beitrag auch die Fussnoten 9 und 12. Wenn im Text auf andere Studien Bezug genommen wird, werden die dort verwendeten Begriffe benutzt.

6 Er zeigt, dass Handeln und Verhalten im Habitus der Menschen gründen. Siehe dazu die Ausführungen weiter unten. 
tensiven Fernseh- und Videokonsum, bringen einen neuen Sozialisationstyp hervor, der in pädagogischen Einrichtungen bestimmte Stufen des sozialen Lernens erst nachholen muss [sic!] und andererseits eine Vielzahl von Störungen der Aufmerksamkeit und Konzentrationsfähigkeit, der Sprach- und Denkentwicklung und des sozialen Verhaltens in den Erziehungsalltag einbringt» (Iben 1990, S. 7). Zahlreiche Studien machen seither auf die ungleichen Möglichkeiten und Fähigkeiten von Eltern unterschiedlicher Schichten und Milieus im Hinblick auf die Förderung ihrer Kinder aufmerksam (vgl. Xyländer u. a. 2008), wie etwa Mansel/ Neubauer (1998) und Lange/ Lauterbach (2000), die auf massive Beeinträchtigungen der Bildungschancen von Kindern aus Familien, die in Armut leben, hinweisen. Nach Forschungen von Grundmann (2000) sind unterstützende Sozialisationsbedingungen vor allem in bildungsnahen Milieus anzutreffen. «In bildungsfernen Milieus hingegen, die immer noch einen Grossteil der bestehenden Sozialisationskontexte bestimmen, wird diese Erziehungsvorstellung zwar angestrebt, kann aber von den Eltern häufig (u.a. aufgrund mangelnden Bildungshintergrunds) nicht eingelöst werden» (Grundmann 2000, S. 97, vgl. auch Aufenanger 2004). Insbesondere die PISA- und IGLU-Debatte hat in diesem Kontext neuerdings für Diskussionsstoff gesorgt und den Blick für sozio-ökonomische Aspekte des Kinderalltags geschärft (vgl. Niesyto 2008).

Gesellschaft heute ist zudem - und dies verschärft die soziale Problematik, da nicht alle Schichten gleichermassen am Wohlstand teilnehmen können - zuvörderst Konsumgesellschaft; sie integriert alle Schichten der Bevölkerung - wenn auch mit durchaus unterschiedlichen Chancen und Konsequenzen. Soziale Systeme organisieren sich zunehmend nach ökonomischen Regeln marktwirtschaftlicher Gesellschaften; darin eingebunden ist auch der mediale Wandel. Er wird dominiert vom Phänomen der Digitalisierung und der Konvergenz der Medien, dem Zusammenwachsen von PC, Internet, Fernsehen und Mobilkommunikation: Gesellschaft und Medien stehen miteinander in einer Wechselbeziehung (vgl. Steinmaurer 2003); es hat sich eine "Medienkulturgesellschaft» entwickelt, die sich in den vergangenen Jahrzehnten infolge eines steigenden Angebots unterschiedlicher Medien, Inhalte und Technologien herausgebildet hat (vgl. Steinmaurer 2003, S. 107). Insbesondere die Dualisierung des Fernsehens und die Einführung neuer Medien wie des Internets mit seinen unterschiedlichen Tools und Anwendungsmöglichkeiten oder des digitalen Fernsehens und die damit verbundene Zunahme interaktiver Angebote kennzeichnen gesellschaftliche Veränderungen auf dem medialen Sektor. So erscheint es derzeit nahezu ausgeschlossen, «ausserhalb der Medien zu leben» (ebd). Gesellschaft definiert sich stärker denn je über die in ihr erzeugte und in ihr zirkulierende Kommunikation.

Nicht zu kommunizieren oder von Kommunikation ausgeschlossen zu sein, das meint auch nicht angemessen kommunizieren zu können, gilt damit nahezu als pathologisches Phänomen, als Devianz. Die Förderung kommunikativer Kompe- 
tenz bzw. von Medienkompetenz (vgl. Baacke 1973) ist daher zu einer zentralen Aufgabe geworden. Sie gilt es im Hinblick auf die partizipatorischen Belange von Menschen in einer von Medien geprägten Gesellschaft ernst zu nehmen und die Erforschung ihrer Bedingungen (zur Behinderung bzw. Förderung) als ein wichtiges Anliegen der (Medien-)Sozialisationsforschung zu betrachten.

\section{Mediensozialisationsforschung - mit Bezug zur Lebenswelt von Heranwachsenden}

Die je spezifische Lebenswelt, in der sich Kinder- und Jugendleben vollzieht und die massgeblich vom sozialen Milieu und der damit zusammenhängenden Lebensführung im Alltag geprägt wird, ist das Fundament der sich täglich aufs Neue vollziehenden Alltagsgestaltung und -bewältigung in den Familien. Ein Rekurs auf die Lebenswelt erscheint daher für Mediensozialisationsforschung bzw. Kinder- und Jugend(medien)forschung unerlässlich; denn die eigenen Deutungsmuster und biographischen Schemata der Betroffenen dürfen nicht ausser Acht gelassen werden. In ihnen spiegeln sich zum einen die Vorstellungsbilder, Interpretationsfolien und Handlungsmodelle zwischen Mensch und Objekt (vgl. Hörning 2001, S. 158.) wider, zum anderen schlagen sich darin kulturell geprägte Gepflogenheiten, Wissensbestände und Kompetenzen, die in die Praktiken und Handlungsmuster einfliessen nieder (vgl. ebd).

Die vielfältigen Bezüge und Besonderheiten im rekursiven Zusammenspiel unterschiedlicher Sozialisationsagenturen mit dem Sozialisanden, dem Kind bzw. Jugendlichen selbst, erschliessen sich im konkreten Einzelfall im Kinder- bzw. Jugendalltag; sie lassen sich in ihrer vollen Tragweite und Komplexität aber nur vor dem lebensweltlichen Hintergrund der Kinder und Jugendlichen verstehen. So erscheint es notwendig, den Blick auf gesellschaftliche Strukturen zu lenken, die die «soziale Rahmung» bedingen. Die Herausforderung in der Mediensozialisationsforschung besteht also darin, sowohl Prozesse auf der Mikro- als auch der Makroebene mitzubedenken und ein Forschungsdesign zu entwickeln, das es erlaubt, diese vielfältigen dynamisch-transaktionalen und molaren (ökologischen) Prozesse auf möglichst integrative und ganzheitliche Weise zu modellieren (vgl. Früh 2002). Dies bedeutet, sowohl die Perspektive auf das Individuum als auch die Gesellschaft zusammenschauend so zu operationalisieren und empirisch fassbar zu gestalten, dass sich nachvollziehen lässt, wie etwa die konkrete Aneignung medialer Symbolangebote durch Kinder und Jugendliche im alltäglichen Umgang mit unterschiedlichen Medien und unterschiedlichen Medienangeboten im Kontext ihrer spezifischen Sozialisationsverläufe tatsächlich von Statten geht. Wie auch Grundmann (2004) deutlich hervorhebt, verfängt sich die Herleitung von Sozialisation aus den manifesten Strukturen des Subjekts oder aus den manifesten Strukturen der sozialen Wirklichkeit «in den Fängen genau jenes Erkenntnisgegenstandes, den es letztlich zu bestimmen gilt» (ebd); der eigentliche Analysegegenstand, eine 
Sozialisationspraxis, «bei der unter der Bedingung fortwährender Um- und Neugestaltung differente Erfahrungen, Interessen und Wertorientierungen vermittelt und ausgetauscht (mithin kommunikativ integriert) werden» (ebd, S. 326), werde daher oft schlicht verfehlt. Eine zwar proklamierte Zusammenschau zentraler Faktoren bzw. eine behauptete, aber nicht angemessen operationalisierte integrative Sichtweise muss scheitern, da sie es nicht schaffen kann, die tatsächlichen Konstitutionsprozess von Beziehungen, die den Sozialisationsprozess tragen, im Zusammenhang der Alltagspraxen und Aktionen der sozialen Akteure in ihren tatsächlichen Lebensverhältnissen zu erfassen und zu beschreiben.

Um die auch für die Sozialisation von Heranwachsenden relevanten Bedingungsgeflechte wissenschaftlich nachvollziehbar machen zu können, gilt es, sowohl die Umweltbedingungen als auch die damit in Interaktion stehenden individuell-subjektiven Bedingungen zu berücksichtigen und dabei sorgfältig auf die Nuancen der «feinen» und oft auch weniger feinen «Unterschiede» zu achten, die in der Sozialisation zum Tragen kommen. Diese hängen zusammen mit dem sozialen Feld, dem Milieu, in dem Kinder ihre Erfahrungen machen - mediale wie nicht-mediale , ihre Identität aufbauen, Handlungskompetenz erwerben, sich selbst und ihre Umwelt beurteilen, bewerten und einzuordnen lernen. So erscheint es in Forschungen zur (Medien-)Sozialisation wichtig, die gesamten lebensweltlichen Hintergründe mit einzubeziehen, um möglichst umfassende Ergebnisse liefern zu können.

Eine solche Betrachtung beugt einer Engführung des Blicks vor, zumal explizit auch die alltägliches Handeln fundierende «Materialität», wie sie Bourdieu in seiner Theorie der Praxis hervorhebt (vgl. Ebrecht/ Hillebrandt 2002, S.11), mit ins Visier genommen wird, also die Lebenswelt in ihren sozioökonomischen sowie molaren (ökologischen) Konstellationen, in der sich die alltägliche Lebensführung konkret vollzieht - und praktisch wird - so wie es auch Dieter Baacke gefordert hat (vgl. z. B. 1988).

\section{Zur Rekonstruktion von Alltag im Kontext der Mediensozialisationsforschung} «Da der Mensch die Realität nur konstruierend erfährt, kann niemals, Realität an sich', sondern nur die Realitätskonstruktion Gegenstand einer empirischen Analyse sein» (Charlton/ Neumann 1988, S. 95). Wissenschaftler sind bei Erklärungen auf Rekonstruktionen von Alltagskontexten und Alltagsstrukturen angewiesen, d.h. sie können ohne das Nachzeichnen des individuellen Handelns in seinem je spezifischen Kontext nichts wirklich über das Handeln des Menschen in seinem Alltag erfahren. Sie müssen sich dazu mit dem Alltagswissen der für eine Untersuchung in Frage kommenden Menschen beschäftigen und es so gut wie möglich verstehen. Der Rekurs auf das Alltagswissen ${ }^{7}$ von Sozialisationsagenten und Heranwach-

Alltagswissen muss von professionellem und wissenschaftlichem Wissen abgegrenzt werden. Wissen allgemein impliziert all "das Wissen um das, was normativ oder funktional vorgegeben ist» (Schweizer 2007, S. 275), aber auch Wissen um Normbildung, Normdurchsetzung, Normerosion, 
senden erscheint notwendig, um zu verstehen, wie sich Sozialisationsprozesse vollziehen. Alltagswissen korrespondiert mit sozialen Praktiken in der Lebenswelt; Alltagswissen näher zu kommen verlangt damit einen aufmerksamen Blick auf die je spezifische Situation, d.h. auf den Menschen in seiner speziellen Lebenssituation. Alltagswissen fliesst in entscheidendem Masse in das lebensweltlich bedingte ,Vorwissen' jedes Einzelnen mit ein, das nunmehr - dies ein Verdienst der «kognitiven (oder auch psychologischen) Wende» seinerzeit in der Psychologie - als Wahrnehmungs- und Informationsverarbeitungsprozess, der sowohl Selektionsals auch Konstruktionsleistungen voraussetzt, begriffen wird. Das ,Vor'- bzw. ,Weltwissen' von Menschen - selbstverständlich auch von Kindern und Jugendlichen - organisiert sich in Form von Schemata, die im Laufe des Sozialisationsprozesses durch aktive Welterfahrung erworben und ausdifferenziert werden. Höhere Organisationsformen von Schemata stellen ,Scripts' und ,Frames' dar. Während ,Frames' Schemata-Zusammenschlüsse zu Gesetzmässigkeiten und Regelhaftigkeiten beschreiben, ist unter einem ,Script' eine Vernetzung von Schemata zu verstehen, die Interaktionsabläufe regeln (vgl. Beaugrande/ Dressler 1981).

In solchen «Spielregeln des sozialen Lebens (,Scripts')» (Charlton/ Neumann 1990, S. 30), die auch geprägt werden von jeweils milieuspezifischen kulturell dominanten Wahrnehmungsschemata und die planvolles Handeln des Menschen ermöglichen, lassen sich die von der Regelförmigkeit des Sozialen mitbestimmten Verhaltens- und Umgangsweisen von Menschen erfassen. ${ }^{8}$ Auf sie gilt es im Kontext der (Medien-)Sozialisationsforschung genau zu achten. Sie zu erfassen, zu verstehen und möglichst exakt zu beschreiben, erscheint als eine zentrale Herausforderung einer Mediensozialisationsforschung, der es darum geht, die Rolle von Medien im Kontext von Sozialisationsprozessen in der Lebenswelt von Menschen nachzuspüren.

Substitute von Nomen, Normalitätsunterstellungen und Normalisierung. «Es ist auch Wissen, wie man mit Normen, Regeln und Ritualen umgeht, welche Entscheidungen anstehen, welche Verfahren gewählt werden können, wie lange die Handlungsketten sind, die man ins Kalkül ziehen muss, welche Lebensstile und Weltbilder, die immer schon da sind, man berücksichtigen muss, wer welchen Handlungsbereich beherrscht und wie das alles - höchst selektiv - von Alltagswirklichkeit als ausgezeichneter Wirklichkeit gedeckt ist» (ebd).

8 Nach der psychologischen Wende setzte sich die Einsicht durch, dass sich die Regelförmigkeit des Sozialen nicht nur als Inhalt konzipiert, dem sich das bereits einsichts- und lernfähige Individuum zuwendet, sondern dass schon die sozialisatorische Interaktion die Struktur sein könnte, in der sich Subjektivität und Einsichtsfähigkeit erst bilden müssen (vgl. Charlton/ Neumann 1990, S. 30). 


\section{Das Beispiel (Medien-)Sozialisationsstudie bei sozial benachteiligten Kindern in Österreich}

\section{Zur Ausgangslage und zur Fragestellung der Studie}

Die Studie zur (Medien-)Sozialisationsprozessen bei Kindern in sozial benachteiligten Milieus ${ }^{9}$ in Österreich (Paus-Hasebrink/ Bichler 2008), die im Folgenden als Beispiel vorgestellt werden soll, versteht sich als eine mit Norbert Elias gesprochene "engagierte Sozialforschung», keinesfalls um eine "distanzierte» (vgl. 1987, vgl. Forster 2008). ${ }^{10}$ Die Studie möchte Aufmerksamkeit für die Belange sozial benachteiligter Kinder und ihren Medienumgang schaffen, um eine Vorlage zur Diskussion von Möglichkeiten und Wegen zu bieten, wie die Bedingungen der sozial benachteiligten Kinder in ihren Familien verbessert und damit Bedingungen für eine möglichst geglückte (Medien-)Sozialisation für diese Kinder geschaffen werden können.

Zwar liegt inzwischen eine Vielzahl an Untersuchungen vor, die sich mit unterschiedlichen Teilaspekten von Sozialisation befassen und das Verhältnis Kinder, Medien und Gesellschaft aus verschiedenen Perspektiven analysieren. Der Medienumgang in sozial benachteiligten Familien wurde aber bisher nicht nur in Österreich selten thematisiert und wissenschaftlich untersucht. So bestand dringender Forschungsbedarf. Dies diente als Ausgangspunkt für die Studie, die den mit dem sozialen Wandel einhergehenden sozialisatorischen Bedingungen speziell für Kinder aus Familien in einer schwächeren sozialen Lage sowie ihrer Bedeutung für den Prozess der (Medien-)Sozialisation nachgeht. Ihre zentrale Forschungsfrage lautet: Welche Relevanz kommt Medien im Laufe des Sozialisationsprozesses bei der Identitätskonstruktion, dem Aufbau von Wissen und in der Wertevermittlung im Vergleich zu anderen Sozialisationsagenten (vor allem der Familie) bei sozial benachteiligten Kindern zu?

Soziale Milieus sind durch grundlegende Anschauungsweisen geprägt, die sie milieuintern teilen. Darin unterscheiden sich soziale Milieus von jeweils anderen sozialen Milieus (vgl. Weiss 1997, S. 259). In sozialen Milieus manifestiert sich der je spezifische lebensweltliche Zusammenhang von Lebenslage (soziale Lage) und Lebensentwurf. Soziale Milieus stellen ein Portrait der sozialen Gliederung und Struktur der Gesellschaft dar (vgl. ebd S. 246). Als Basis der Auswahl von Kindern bzw. Familien aus sozial benachteiligten Milieus dienten relevante Faktoren zur Bestimmung der sozialen Lage der Probanden; siehe dazu Fussnote 11.

10 Es geht bei dieser Art Forschung aber nicht etwa, wie Forster hervorhebt, um eine subjektiv bestimmte Forschung (vgl. 2008, S. 51), sondern um eine Sozialforschung, die ein wissenschaftliches und gleichzeitig ein soziales Anliegen hat. 
Folgende Fragen auf drei Ebenen standen dabei im Mittelpunkt:

- Wie beeinflussen die gesellschaftlich-strukturell bedingten Faktoren der sozialen Benachteiligung den Bereich der Handlungsoptionen der Kinder, ihrer Geschwister und ihrer Eltern in ihren Familien?

- Welche Handlungsentwürfe lassen sich bei den Kindern und ihren Familien eruieren? Das heisst: Welche subjektiven Wahrnehmungen sozio-ökonomischer Faktoren, also der sozialen Lage, lassen sich im Zusammenspiel der Verzahnung von Praxis und handlungsleitender Anschauung bei den Kindern und ihren Eltern in ihren Familien erkennen? Und verbunden damit: Welche Rolle spielt der jeweilige sich aus diesem Zusammenspiel entwickelnde ,Eigensinn' der Kinder, ihrer Eltern und Geschwister bzw. der Familie als ganzes?

- Welche jeweiligen Handlungskompetenzen können dabei die Kinder, ihre Eltern und Geschwister in ihren Familien entwickeln? Welche subjektiven Ausprägungen des Konglomerats aus Handlungsoptionen und der vor diesem Hintergrund möglichen Handlungsentwürfe lassen sich auf der Handlungsebene zur sinnvollen Alltagsgestaltung identifizieren? Wie sehen also die Handlungsweisen im Alltag der sozial benachteiligten Kinder, ihrer Eltern und Geschwister in ihren Familien im Umgang mit Medien unter den jeweiligen gesellschaftlichstrukturellen Bedingungen ihres Lebensumfelds, also in ihrem sozialen Raum, aus?

\section{Zur theoretischen Konzeption der Studie}

Im Mittelpunkt steht ein Konzept integrativer Mediensozialisationsforschung. Es dient als theoretisches Fundament für eine auf drei Jahre angelegte qualitativen Panelstudie mit insgesamt 20 Familien; ihr Ziel war es, Bedeutungsstrukturen und subjektiven Bedeutungszuweisungen von Kindern und Eltern im Umgang mit Medienangeboten nachzugehen und Zusammenhänge im Zusammenspiel von unterschiedlichen Faktoren, die das Leben sozial benachteiligter Kinder, mithin ihre Sozialisation prägen, zu erkennen und zu beschreiben. Im Rahmen der Auswahl kam zur Bestimmung der sozialen Lage ${ }^{11}$ die Kombination der Lebensbedingungen

11 Wie Hradil betont, sind mit vielen sozialen Positionen Lebens- und Arbeitsbedingungen verknüpft, «die ihre Träger gegenüber anderen nicht einfach als in bestimmter Hinsicht unterschiedlich [...] erscheinen lassen, sondern gleichzeitig auch als besser- oder schlechter-, höher- oder tiefergestellt, bevorrechtigt oder benachteiligt [...]» (Hradil 1999, S. 11). Diese teils hierarchischen Statusunterscheidungen bzw. -verteilungen, die sich auf unterschiedliche Dimensionen beziehen können, bezeichnen soziale Ungleichheit. Wegen der hohen Komplexität sozialer Lebenslagen wird allerdings zunehmend davon Abstand genommen, eine einfache schicht- und klassenspezifische Teilung der Bevölkerung vorzunehmen; denn Merkmale sozialer Ungleichheit ergeben sich nicht allein aus klassischen berufsbedingten Dimensionen, wie etwa Einkommen und Prestige, sie resultieren vielmehr aus mehreren unterschiedlichen Dimensionsebenen, also einem mehrdimensionalen Bedingungsgefüge (vgl. Hradil 1999). Hradil schlägt daher den Begriff «soziale Lage» vor; soziale Lagen sind grundsätzlich durch primäre, sekundäre und weitere Lebensbedingungen charakterisiert und begründen «typische Kontexte von Handlungsbedingungen, die vergleichsweise gute oder schlechte Chancen zur Befriedigung allgemein anerkannter Bedürfnisse gewähren» (Hradil 1987, S. 153). Das 
Armut bzw. Armutsgefährdung sowie niedrige Bildung zum Tragen; mitbedacht wurden die Wohnregion der Familie, ob Stadt oder Land, das Geschlecht sowie das für die Panelstudie relevante Alter der Kinder. Bei der Auswertung wurden alle Familien zum einen in einem fokussierten Analyseschritt im Hinblick auf zentrale Kategorien, wie z.B. ihre Mediennutzung, ihre Freizeitgestaltung sowie die (Medien)Erziehungskonzepte der Eltern, hin beleuchtet; im daran anschliessenden kontextuellen Auswertungsteil wurde zum anderen jeder Einzelfall für sich zusammenhängend daraufhin analysiert, wie und unter welchen Bedingungen bzw. mit welcher Motivation die Probanden mit Medien umgehen, wie also Eltern und speziell die im Mittelpunkt der Studie stehenden Kinder Mediensymbolik auswählen und einsetzen, um ihrem Leben Sinn zu verleihen und die jeweiligen Herausforderungen in ihrem ganz spezifischen Alltag zu bearbeiten.

Ausgehend von der Frage nach dem je ,praktischen Sinn' der Umgangsweisen von Kindern und Eltern in ihren Familien mit Medien gilt anhand der fünf Begriffe Soziales Milieu - Habitus - Erleben - Orientierung - Identität (siehe auch PausHasebrink 2005) der Blick den jeweiligen Möglichkeiten der Kinder aus sozial benachteiligten Familien, Identität auszubilden und Handlungskompetenz zu erwerben. Als Ausgangspunkt der Überlegungen der theoretischen Konzeption diente Bourdieus «Theorie der Praxis» (vgl. 1979), um das soziale Feld, in dem jeweils bestimmte Handlungsziele und bestimmte Ressourcen wirksam werden sowie bestimmte Handlungsmuster jeweils «am Platz» (Weiss 2000, S. 47) sind, umreissen zu können. Dabei geht es um die sozialen Räume oder, um einen Begriff von Bourdieu einzuführen, um das soziale Milieu, in dem sich im vorliegenden Fall die Kinder aus sozial benachteiligten Lebensverhältnissen und ihre Eltern bewegen und aufwachsen. Mit dem Blick auf das Soziale Milieu und auf seine phänomenologische Ausprägung, den Habitus, wird ein zentraler Lebensgrund reflektierbar, die Verflochtenheit der genannten Aspekte kann besser erfasst werden.

Habitus ist demnach also das «Vermittlungsglied zwischen der Stellung im sozialen Raum und dem für die jeweilige Position typischen Lebensstil, den Praktiken und Vorlieben, die von einer Person in dieser Stellung erwartet werden. Er bestimmt die Praxis, auch wenn sich die Handelnden dieser Wirksamkeit des Habitus für ihre soziale Wahrnehmung und ihr konkretes Handeln nicht oder nur selten bewusst sind» (Baumgart 1997, S. 201f.). Je nach Milieuverortung des Individuums

Konzept der sozialen Lagen verhindert, dass soziale Ungleichheit bzw. im konkreten Fall soziale

Benachteiligung zu eindimensional definiert wird, d. h. zu sehr auf die Berufstätigkeit und die vertikale Ebene sozialer Benachteiligung konzentriert wird. Nach Hradil (1999, S. 27f. und 30) lassen sich folgende Dimensionen zur Bestimmung der sozialen Lage identifizieren: Objektive Dimensionen: Einkommen, Bildung, Arbeits-, Wohn-, Umwelt- und Freizeitbedingungen, Geschlecht, Alter (und Kohortenzugehörigkeit), Beruf, ethnische Zugehörigkeit (Nationalität), Familienverhältnisse sowie in Bezug auf subjektive Dimensionen: Integration, Prestige (=Ansehen eines Gesellschaftsmitglieds), sozial begründete Macht (= Ausmass an Handlungsfreiheit). Für die im Kontext der Studie notwendige Konstruktion von Auswahlvariablen zur «sozialen Benachteiligung» dienten mit Bezug auf Hradil die zentralen charakteristischen Merkmale «sozialer Ungleichheit», eine Dimensions-Kombination der Lebensbedingungen Armut und niedrige Bildung. 
gelten demnach bestimmte Regeln, die beherrscht werden müssen, um innerhalb dieses Status anerkannt zu werden. «Wie wir uns kleiden, was wir lesen, wie wir unsere Wohnungen einrichten, was wir für ein gutes Essen halten - dies alles liegt nicht nebeneinander, sondern weist einen einheitsstiftenden Stil auf, der unsere Zugehörigkeit zu einer bestimmten gesellschaftlichen Gruppe kenntlich macht. Der Habitus ist die vermittelnde Instanz zwischen subjektiven und objektiven Dimensionen sozialer Existenz. Er fungiert wie die Grammatik unserer Sprache. Wir benutzen sie, aber wir wenden sie nicht bewusst von Fall zu Fall an» (Baumgart 1997, S. 202). Der Habitus kann also als das Prinzip verstanden werden, nach dem die Methoden der Lebensführung und darin verwoben die alltagskulturellen Praxen von Menschen entfaltet werden, die, je nach sozialem Milieu, ihren Ausdruck in unterschiedlichen Lebensstilen ${ }^{12}$ finden (vgl. Weiss 1997, S. 246). Angehörige eines Milieus stellen demnach Gemeinsamkeiten (bewusst oder unbewusst) her, indem sie Dingen und Ereignissen ähnliche Bedeutung zuschreiben und Erlebtes ähnlich interpretieren.

Als Ausgangspunkt weiterer Überlegungen diente die Erkenntnis, dass Gemeinschaft in geteilten Bedeutungen und selbstverständlichen lebensgeschichtlichen Praktiken gelebt wird (vgl. ebd). Diese stellen sich im gemeinsamen Erleben her, sind eingelagert in gemeinsame Gewohnheiten. Gemeint sind damit auch die «körperlich eingeschriebenen», «nicht gedachten» Kategorien, die im Begriff des Habitus miterfasst und als "gemeinschaftliches Wissen» bezeichnet werden können (vgl. Lash 1996, S. 268). Erleben (der Begriff «Erlebnisgesellschaft» weist darauf hin) wird - unabhängig vom sozialen Milieu, wenn auch mit je spezifischer das Milieu charakterisierender Ausprägung - in den jeweils favorisierten unterschiedlichen Erlebnisformen und Erlebnisweisen von Erwachsenen und Kindern, in Familien und Peer-Groups grossgeschrieben.

Welche Rolle spielen dabei Medien? Nicht selten gewinnen heute kommerzielle, häufig crossmedial vermarktete populärkulturelle Phänomene, wie Helden und Heldinnen aktueller Fernsehserien und Computerspiele gerade für Kinder eine grosse Rolle. Sie bieten Orientierung und helfen Heranwachsenden (wie auch Erwachsenen), ihren Platz im Alltag zu finden. Insgesamt sind Orientierungsmuster kulturell tradiert; sie verweisen auf den gesellschaftlichen Standort und steuern, zu Alltagswissen geronnen, das individuelle Handeln von Menschen mit. Sie helfen, soziale und personale Identität auszubilden, also den eigenen Standort zu finden, Handlungskompetenz zu erlangen.

Mit dem Blick aber auf die Methoden der Sinngebung, das Tableau alltagspraktischer Orientierungsmuster, d. h. wie Menschen ihren Handlungen im Alltag Sinn

12 Dies bedeutet, dass in diesem Kontext der Begriff «Lebensstil» breiter zu verstehen ist und nicht gleichgesetzt werden darf mit dem Begriff für «expressive» Momente der Lebensführung, die sich in der Warenwelt und der Kulturindustrie entnommenen «Stilfragmente» manifestieren und die ihren Niederschlag in Lifestyle-Typologien der Marktforschung finden. Derartige Stilisierungen sind der Mode unterworfen und rasch vergänglich (vgl. Weiss 1997, S. 245ff.). 
geben, stellte für die vorliegende Studie nicht länger die Frage entweder aus einer ,subjektiven' oder einer ,objektiven' Perspektive. Eine derartige Dichotomie findet sich auch heute noch weitgehend in der modernen Sozialisationsforschung, die auf die von Klaus Hurrelmann eingeführten Begriffe, innere Realität' und,äussere Realität' zurückgehen (Hurrelmann/ Ulich 1991a; b): Auf der einen Seite, der ,inneren Realität', stehen die subjektiven Faktoren, auf der anderen, der ,äusseren Realität', ist von den gesellschaftlich-strukturellen Bedingungen, den so genannten ,objektiven' Faktoren, die Rede. Ausgangspunkt der Studie «(Medien-Sozialisation) bei sozial benachteiligten Kindern» war vielmehr die Perspektive des "praktischen Sinns», mithin der ,Praxeologie'. Dabei wird nach wie vor das je individuelle, aber dennoch über die subjektive Repräsentation hinaus weisende Lebensumfeld und die jeweilige darin eingelagerte Lebensführung ins Visier gefasst, mithin das soziale Milieu, also die sozialen Räume, die dem Einzelnen, den Eltern sowohl als auch den Kindern und Jugendlichen wie ihren Peer-Groups, tatsächlich oder symbolisch zur Verfügung stehen, den Räumen also, in denen der Einzelne seine ,Kapitalien' möglichst sinnvoll einsetzt; denn auch bereits junge Kinder streben danach, ihrem Leben Sinn zu verleihen, nicht selten gewinnt gerade dabei Mediensymbolik eine hohe Bedeutung. Die Grundkoordinaten der Sozialisation ihrer Kinder bestimmen jedoch die Eltern mit ihrer Lebensführung, mit ihrer je spezifischen Antwort auf die sozialen Bedingungen ihres sozialen Raumes.

Im Zentrum der «Theorie der Praxis», mithin des praktischen Sinns, steht der ,Eigennutz' und entsprechend dazu das ,Taxieren'. Es geht also darum zu taxieren, wie man Chancen in den von Habermas benannten Sphären alltagspraktischen Handelns wie Erwerbsleben (Arbeit, Verdienst, Vermögen), Politik und Recht (gesellschaftliche Ordnung, Recht, Moral) und Privatleben (Liebe, Beziehung, Glück) (vgl. Habermas 1988, S. 473) nutzen kann, um eine individuelle Erfolgsperspektive zu gewährleisten. ${ }^{13}$

Der Einzelne, auch bereits ein Kind oder ein Jugendlicher, legt sein praktisches Handeln als einen Versuch an, kraft seiner ,Kapitalien' die ins Auge gefassten Chancen des jeweiligen sozialen Ortes zu verwirklichen. Dies gilt in besonderer Weise jedoch für die Eltern. Dabei ist im Auge zu behalten, dass es sich keinesfalls um einen klar bewussten Vorgang handelt, sondern um einen komplexen, von vielen Faktoren, wie etwa der formalen Bildung, dem Geschlecht und damit verbundener Körperlichkeit des je Einzelnen mitbestimmten Prozess. Aufmerksam gilt es dabei im Blickfeld zu behalten, dass der 'Schatz der subjektiven Kapitalien' ungleich verteilt ist und auch mit dem jeweiligen Ort zusammenhängt, in dem sich der Einzelne bzw. eine Familie bewegt.

\footnotetext{
13 Ben Bachmair weist mit Blick auf die Sendung Popstars (2006) darauf hin, dass diese Handlungsmuster als Orientierungsvorlagen für Konkurrenz und Kooperation anbieten und Hinweise darauf, was man für Erfolg und Startum leisten muss.
} 
Daher steht im Zentrum der Frage nach dem je ,praktischen Sinn' die Frage nach den unterschiedlichen sozialen Milieus und ihren Habitusausprägungen; das heisst nach den unterschiedlichen Möglichkeiten des Einzelnen oder einzelner Gruppen, wie im vorliegenden Fall einer Familie bzw. der Familien aus sozial benachteiligten Milieus, Identität auszubilden und Handlungskompetenz zu erwerben. Mit dem Blick auf den ,praktischen Sinn', die Praxeologie, kann es gelingen, eine Brücke zwischen sog. ,subjektiven' und ,objektiven' Faktoren zu schlagen und psychologische und soziologische Ansätze, die die (Medien-) Sozialisationsforschung prägen, zu vereinen.

Im Kontext der Methoden der Sinngebung, des praktischen bzw. alltagspraktischen Sinns, kann nunmehr die sich jeweils spezifisch vollziehende Entwicklung folgender drei Bereiche zusammen gedacht werden:

- der Bereich der Handlungsoptionen. Darunter sind die gesellschaftlich-strukturell bedingten Faktoren zu verstehen,

- der Bereich der Handlungsentwürfe. Sie stellen die subjektiven Wahrnehmungen dieser Struktur im Zusammenspiel der Verzahnung von Praxis und handlungsleitender Anschauung dar, verbunden mit dem jeweiligen sich aus diesem Zusammenspiel entwickelnden ,Eigensinn' des Einzelnen bzw. einzelner Familien und

- der Bereich der jeweiligen Handlungskompetenzen. Darunter sind die subjektiven Ausprägungen dieses Konglomerats, also sowohl der Handlungsoptionen und der vor ihrem Hintergrund möglichen Handlungsentwürfe, auf der Handlungsebene zur sinnvollen Alltagsgestaltung zu verstehen, was das Kind, der Jugendliche, seine Eltern bzw. die Familie im Zusammenspiel also tatsächlich von seinen bzw. ihren Handlungsentwürfen unter den jeweiligen gesellschaftlich-strukturellen Bedingungen in ihrem Lebensumfeld, ihrem sozialen Raum in der Lage ist umzusetzen.

Mit einem derartigen Ansatz ist der Schritt über eine allzu häufige Verengung des Blickwinkels hinaus getan, sowohl über die entweder gesellschaftlich-strukturelle Ebene als auch die Ebene der Handlungsentwürfe und Handlungskompetenzen und die subjektiven Umgangsweisen von Kindern bzw. Jugendlichen, ihren Eltern und der Familie. Der Blick auf die Methoden der Sinngebung, und damit auch auf das soziale Milieu, bietet die Möglichkeit, dem induktiven Schluss aus einer zu stark verfeinerten subjektiven Perspektive zu entgehen; diese führt zur Beschreibung subkultureller Szenebildungen oder auch vor allem ästhetisch geprägter Lebensstilorientierungen. Wichtig ist der Schritt hin zu einer Zusammenschau aller wichtigen Faktoren in der (Medien-)Sozialisation Heranwachsender.

Diese steht mit der Rekonstruktion des Sinns, den Eltern und Kinder als Familie und jeweils für sich vor dem Hintergrund ihres sozialen Milieus einzelnen Vorgän- 
gen in ihrer Umgebung, zu geben suchen, um ihre persönliche und soziale Identität so gut wie möglich zu sichern bzw. auszubauen, ihr Kohärenz zu geben und sie für sich erlebbar zu gestalten, im Mittelpunkt der angesprochenen Studie. Dabei geht es darum, der Lebensführung sozial benachteiligter Familien mit Blick jeweils auf den Einzelfall nachzuspüren und Merkmale zu identifizieren, die ein möglichst kohärenten Bild des Lebens von Kindern im Hinblick auf ihren jeweiligen ganz spezifischen (Medien-)Sozialisationsprozess erlauben, den sie jeweils in ihren Familien erfahren.

Vor diesem Hintergrund lassen sich die Gemeinsamkeiten und Unterschiede in den je spezifischen Ausprägungen der Lebensführung von Familien - Kinder und Eltern - aus sozial benachteiligten Milieus identifiziert und Einflussfaktoren und Einflusskonstellationen dingfest machen, die der Mediensozialisation im Kontext des Sozialisationsprozesses von Kindern das je spezifische Gepräge verleihen. So konnten in der Studie - um auch an dieser Stelle einen kleinen Einblick in die Ergebnisse zu bieten - Merkmale identifiziert werden, die die (Medien-)Sozialisation aller Kinder in den Familien der Panelstudie charakterisieren, wie etwa die grosse Bedeutung, die Medien insgesamt für sie gewinnen, sowie ein ähnliches (Medien-) Erziehungsverhalten der Eltern, das vor allem durch Unsicherheit und mangelnde Konsequenz geprägt war (siehe dazu Paus-Hasebrink/ Bichler 2008). Zum anderen liess sich auch deutlich zeigen, dass jedes Kind seine ganz spezifische (Medien-) Sozialisation erfährt und soziale Benachteiligung nicht gleich soziale Benachteiligung ist, sondern individuell bedeutsame Faktoren eines Kindes, wie z. B. seine Begabung oder seine ganz spezifische Antwort auf die Lebensbedingungen in seiner Familie, die Sozialisation mitprägen. Diese Faktoren gewinnen Gewicht im Zusammenspiel mit sozio-strukturellen Veränderungen, etwa mit dem Verlust des Arbeitsplatzes und dem damit verbundenen Umzug einer Familie in eine preiswertere Wohngegend oder mit einer besseren finanziellen Ausstattung der Familie aufgrund einer neuen Partnerschaft der Mutter (vgl. ebd).

\section{Fazit}

Mediensozialisationsforschung, dies war Anliegen des vorliegenden Beitrags, bedarf des Blicks auf die je spezifische Lebenswelt Heranwachsender, die von ihrer sozialen Lage als sozio-strukturelle Basis ihres durch grundlegende Anschauungsweisen massgeblich geprägten sozialen Milieus bestimmt wird. Soziale Hintergründe, vor denen sich das Aufwachsen von Kindern und Jugendlichen vollzieht, müssen in die Forschung miteinbezogen werden. Um die Bedeutung, die Mediensymbolik im Alltag von Heranwachsenden einnimmt, im Kontext untersuchen zu können, erscheint eine integrative sowie interdisziplinäre Forschung notwendig. In einer qualitativ angelegten österreichischen Panelstudie über drei Jahre zur (Medien-)Sozialisation sozial benachteiligter Kinder, deren theoretische und methodologische Fundierung im Beitrag vorgestellt wurde, stand die Frage im Mit- 
telpunkt, welche Relevanz Medien im Laufe des Sozialisationsprozesses bei der Identitätskonstruktion, dem Aufbau von Wissen und in der Wertevermittlung im Vergleich zu anderen Sozialisationsagenten (vor allem der Familie) bei Kindern aus sozial benachteiligten benachteiligten Familien zukommt. Dazu wurde das Forschungsfeld Mediensozialisation theoretisch und forschungspraktisch anhand der fünf Begriffe Soziales Milieu - Habitus - Erleben - Orientierung - Identität mit Blick auf den «praktischen Sinn» menschlicher Handlungsweisen neu aufgespannt und die Relevanz einer molaren (ökologischen) Sichtweise mit dem Fokus auf die Lebensführung in den Familien betont.

Ziel war es, ein Konzept vorzustellen, das es erlaubt, die in der Mediensozialisation relevanten zentralen Ebenen im Kontext zu erfassen: den Bereich der Handlungsoptionen, das heisst die gesellschaftlich-strukturell bedingten Faktoren des Aufwachsens, den Bereich der Handlungsentwürfe also die subjektiven Wahrnehmungen dieser Struktur in der Verzahnung von Praxis und handlungsleitender Anschauung im Zusammenspiel mit dem jeweiligen ,Eigensinn' des Kindes bzw. der Familie sowie den Bereich der jeweiligen Handlungskompetenzen des Einzelnen. So können die sozio-strukturell durch die soziale Lage der Familien mitbedingten Handlungsoptionen vor dem Hintergrund ihrer möglichen Handlungsentwürfe auf der Handlungsebene untersucht werden. Auf ihr vollziehen sich die in die Alltagsgestaltung integrierten Medienumgangsweisen von Heranwachsenden in ihren Familien. Es geht also darum, was ein Kind bzw. ein Jugendlicher im Zusammenspiel mit seinen jeweiligen Sozialisationsakteuren im Kontext des sozialen Milieus, in dem es bzw. er aufwächst, von seinen Handlungsentwürfen umsetzen kann. Gefragt wird dabei auch explizit nach der Rolle von Medienangeboten im Alltag des Heranwachsenden.

\section{Literatur}

Aufenanger, Stefan. «Konzeptionelle Überlegungen zu medienpädagogischen Handreichungen für Eltern, Erzieherinnen und Grundschullehrerinnen.» Medienkindheit - Markenkindheit. Untersuchungen zur multimedialen Verwertung von Markenzeichen für Kinder. Hrsg. v. Ingrid Paus-Hasebrink, Klaus NeumannBraun, Uwe Hasebrink u. Stefan Aufenanger. München: Kopaed, 2004. 265280.

AWO. "Gute Kindheit - Schlechte Kindheit? Armut und Zukunftschancen von Kindern und Jugendlichen in Deutschland. Zusammenfassung der Ergebnisse und Schlussfolgerungen für Politik, Wissenschaft und Praxis zur 1. AWO-ISS-Studie von 1997 bis 2000.»ISS-Pontifex 4/2000: http://www.awo.org/pub/soz_pol/ armut/forsch_ber.html/iss-b-zusammen.pdf (10.12.2007).

Baacke, Dieter. Kommunikation und Kompetenz. Grundlegung einer Didaktik der Kommunikation und ihrer Medien. München: Juventa, 1973. 
Baacke, Dieter. «Sozialökologische Ansätze in der Jugendforschung.» Handbuch der Jugendforschung. Hrsg. v. Heinz-Hermann Krüger. Opladen: Leske + Budrich, 1988. 71-94.

Bachmair, Ben. "Media socialisation and the culturally dominant mode of representation. On the way from the coherent media to semiotic spaces. The example of Popstars." MedienPädagogik. Zeitschrift für Theorie und Praxis der Medienbildung. (2006): http://www.medienpaed.com/2006/bachmair0606.pdf (27.07.2008).

Baumgart, Franzjörg. Theorien der Sozialisation. Erläuterungen. Texte. Arbeitsaufgaben. Bad Heilbrunn: Klinkhardt, 1997

Beaugrande, Rober de/ Dressler, Wolfgang U. Einführung in die Textlinguistik. Tübingen: Niemeyer Verlag, 1981.

Bernstein, Basil. «Social Structure, Language and Learning." Educational Research Vol. 3, Nr. 3 (1961):163-176.

Bourdieu, Pierre. Entwurf einer Theorie der Praxis - auf der ethnologischen Grundlage der kabylischen Gesellschaft. Frankfurt/Main: Suhrkamp, 1979.

Bourdieu, Pierre. Die feinen Unterschiede. Kritik der gesellschaftlichen Urteilskraft. Frankfurt/Main: Suhrkamp, 1982.

Bundesministerium für Familie, Senioren, Frauen und Jugend. Zwölfter Kinderund Jugendbericht. Bericht über die Lebenssituation junger Menschen und die Leistungen der Kinder- und Jugendhilfe in Deutschland. (2005): http://www. bmfsfj.de/ doku/kjb/data/download/kjb_060228_ak3.pdf (21.12.2007).

Burzan, Nicole. Soziale Ungleichheit. Eine Einführung in die zentralen Theorien. Wiesbaden: Verlag für Sozialwissenschaften, 2004.

Charlton, Michael; Neumann, Klaus. «Mediensozialisation im Kontext. Der Beitrag des Kontextualismus und der Strukturanalyse für die Medienforschung.» Publizistik 33. Jg., Heft 2-3 (1988): 297-315.

Charlton, Michael; Neumann, Klaus. Medienrezeption und Identitätsbildung. Kulturpsychologische und kultursoziologische Befunde zum Gebrauch von Massenmedien im Vorschulalter. Schriftenreihe ScriptOralia Bd. 28. Tübingen: Narr, 1990.

Ebrecht, Jörg; Hillebrandt, Frank. «Einleitung: Konturen einer soziologischen Theorie der Praxis.» Bourdieus Theorie der Praxis. Erklärungskraft - Anwendung - Perspektiven. Hrsg. v. Jörg Ebrecht u. Frank Hillebrandt. Opladen: Westdeutscher Verlag, 2002. S. 7-16.

Elias, Norbert. Engagement und Distanzierung. Arbeiten zur Wissenssoziologie. Frankfurt/Main: Suhrkamp, 1987.

Forster, Edgar. "Die Singularität qualitativer Forschung.» Quantitative und qualitative Aspekte. Zu ihrer Komplementarität in der erziehungswissenschaftlichen Forschung. Hrsg. v. Franz Hofmann, Claudia Schreiner u. Josef Thonhauser. Münster/ News York/ München/ Berlin: Waxmann, 2008. 45-55. 
Fromme, Johannes. «Mediensozialisation und Medienpädagogik: zum Verhältnis von informellem und organisiertem Lernen mit Computer und Internet.» Medienpädagogik in der Kommunikationswissenschaft. Positionen, Perspektiven, Potenziale. Hrsg. v. Ingrid Paus-Haase, Claudia Lampert u. Daniel Süss. Wiesbaden: Westdeutscher Verlag, 2002. 155-168.

Früh, Werner. Unterhaltung durch das Fernsehen. Eine molare Theorie. (Unter Mitarbeit von Anne-Katrin Schulze; Carsten Wünsch). Konstanz: UVK Verlagsgesellschaft mbH, 2002.

Geulen, Dieter. «Ungelöste Probleme im sozialisationstheoretischen Diskurs.» Sozialisationstheorie interdisziplinär. Aktuelle Perspektiven. Hrsg. v. Dieter Geulen u. Hermann Veith. Stuttgart: Lucius \& Lucius, 2004. 3-20.

Grundmann, Matthias. «Kindheit, Identitätsentwicklung und Generativität.» Kinder in Familie und Gesellschaft zu Beginn des 21sten Jahrhunderts. Hrsg. v. Andreas Lange $u$. Wolfgang Lauterbach. Stuttgart: Lucius \& Lucius, 2000. 87-104.

Grundmann, Matthias. «Intersubjektivität und Sozialisation.» Sozialisationstheorie interdisziplinär. Aktuelle Perspektiven. Hrsg. v. Dieter Geulen u. Hermann Veith. Stuttgart: Lucius \& Lucius, 2004. 317-346.

Habermas, Jürgen. «Individuierung durch Vergesellschaftung. Zu George Herbert Meads Theorie der Subjektivität.» Nachmetaphysisches Denken. Philosophische Ansätze. v. Jürgen Habermas. Frankfurt/Main: Suhrkamp, 1988. 187241.

Heitmeyer, Wilhelm. Rechtsextreme Orientierungen bei Jugendlichen. Empirische Ergebnisse und Erklärungsmuster politischer Sozialisation. Weinheim/München: Juventa, 1987.

Hörning, Karl H. Experten des Alltags. Die Wiederentdeckung des praktischen Wissens. Weilerswist: Velbrück, 2001.

Hoffmann, Dagmar. «Plädoyer für eine integrative Mediensozialisationstheorie.» Mediensozialisationstheorien. Neue Modelle und Ansätze in der Diskussion. Hrsg v. Dagmar Hoffmann u. Lothar Mikos. Wiesbaden: VS Verlag, 2007. 1126.

Hradil, Stefan. Sozialstrukturanalyse in einer fortgeschrittenen Gesellschaft. Von Klassen und Schichten zu Lagen und Milieus. Opladen: Leske+Budrich, 1987.

Hradil, Stefan. Soziale Ungleichheit in Deutschland. Opladen: Leske+Budrich, 1999.

Hugger Paul. «Vielfalten von Kindheiten - eine wie heute.» Kind sein in der Schweiz. Eine Kulturgeschichte der frühen Jahre. Hrsg. v. Paul Hugger. Zürich: Offizin, 1998. 33-35.

Hurrelmann, Klaus; Ulich, Dieter (Hrsg.) Neues Handbuch der Sozialisationsforschung. 4., völlig neu bearb. Aufl. Weinheim/Basel: Beltz Verlag, 1991a.

Hurrelmann, Klaus; Ulich, Dieter. "Gegenstands- und Methodenfragen der Sozialisationsforschung.» Neues Handbuch der Sozialisationsforschung. 4., völlig neu 
bearb. Aufl. Hrsg. v. Klaus Hurrelmann u. Dieter Ulich. Weinheim/Basel: Beltz Verlag, 1991b. 3-20.

Iben, Gerd (Hrsg.). Erzieheralltag. Situatives Arbeiten mit sozial benachteiligten Kindern. Überarb. Neuaufl. Mainz: Matthias-Grünewald-Verlag, 1990.

Kohn, Melvin L.. Class and Conformity. A Study in Values. Homewood: Dorsey Press, 1969.

Kränzl-Nagl, Renate; Mierendorff, Johanna. «Kindheit im Wandel - Annäherungen an ein komplexes Phänomen.» Sozialwissenschaftliche Gesellschaft: SWS Rundschau Schwerpunktheft Lebenswelten von Kindern 47. Jg., Heft 1, (2007): 3-25.

Lange, Andreas. "Theorieentwicklung in der Jugendforschung durch Konzeptimport. Heuristische Perspektiven des Ansatzes "Alltägliche Lebensführung.» Theoriedefizite der Jugendforschung. Standortbestimmung und Perspektiven. Hrsg. v. Jürgen Mansel, Hartmut Griese u. Albert Scherr. Weinheim: Juventa, 2003. 102-118.

Lange, Andreas; Lauterbach, Wolfgang (Hrsg.). Kinder in Familie und Gesellschaft zu Beginn des 21. Jahrhunderts. Stuttgart: Lucius \& Lucius, 2000.

Lash, Scott. "Reflexivität und ihre Doppelungen». Reflexive Modernisierung. Eine Kontroverse. Hrsg. v. Ulrich Beck, Anthony Giddens u. Scott Lash. Frankfurt: Suhrkamp, 1996. 195-286.

Niesyto, Horst. Medienkulturen und soziale Ungleichheit - Einführung in die Thematik. Vortrag im Rahmen der Arbeitsgruppe «Medienkulturen und soziale Ungleichheit. Zum Spannungsfeld von kulturtheoretischer und lebenslagenbezogener Medienforschung. In memoriam Dieter Baacke; 21. Kongress der Deutschen Gesellschaft für Erziehungswissenschaft (DGfE) vom 16. - 19. März 2008 in Dresden (unveröffentlichtes Vortragsmanuskript).

Palentien, Christian. «Armut - Subjekt - Sozialisation. Ein Plädoyer für eine Stärkung sozialisationstheoretischer Perspektiven in der aktuellen Diskussion eines sozialen Phänomens.» Theoriedefizite der Jugendforschung. Standortbestimmung und Perspektiven. Hrsg. v. Jürgen Mansel, Hartmut M. Griese u. Albert Scherr. Weinheim und München: Juventa Verlag, 2003. 91-101.

Paus-Haase, Ingrid. «Zur Bedeutung von Medienhelden in Kindergarten, PeerGroups und Kinderfreundschaften. Eine Untersuchung zum Umgang von Vorschulkindern mit Mediensymbolik im lebensweltlichen Zusammenhang.» Rundfunk und Fernsehen 47. Jg., Heft 1 (1999): 5-24.

Paus-Hasebrink, Ingrid. "Grundsätzliche Überlegungen zu ,neuen Denkwerkzeugen' in der Jugend(medien)forschung.» Perspektiven der Medienpädagogik in Wissenschaft und Bildungspraxis. Hrsg. v. Hubert Kleber. München: Kopaed Verlag, 2005. 74-85. 
Paus-Hasebrink, Ingrid; Bichler, Michelle. Mediensozialisationsforschung - Theoretische Fundierung und Fallbeispiel sozial benachteiligte Kinder. Wien/Innsbruck: Studienverlag, 2008. (im Druck).

Spanhel, Dieter. Handbuch Medienpädagogik. Bd.3: Medienerziehung. Stuttgart: Klett-Cotta, 2006.

Schweizer, Herbert. Soziologie der Kindheit. Verletzlicher Eigen-Sinn. Wiesbaden: VS Verlag, 2007.

Soeffner, Hans-Georg. "Kulturmythos und kulturelle Realität(en).» Kultur und Alltag. Hrsg. v. Hans-Georg Soeffner. Göttingen: Schwartz, 1988. 3-20.

Sutter, Tilmann. «Sozialisation als Konstruktion subjektiver und sozialer Strukturen. Aktualität und künftige Perspektiven strukturgenetischer Sozialisationsforschungen.» Sozialisationstheorie interdisziplinär. Aktuelle Perspektiven. Hrsg. v. Dieter Geulen u. Hermann Veith. Stuttgart: Lucis \& Lucius, 2004. 93-115.

Sutter, Tilmann. «Zur Bedeutung kommunikativer Aneignungsprozesse in der Mediensozialisation.» Mediensozialisationstheorien. Neue Modelle und Ansätze in der Diskussion. Hrsg. v. Dagmar Hoffmann, Lothar Mikos. Wiesbaden: VS Verlag, 2007. 131-145.

Statistik Austria (Hrsg.). Einkommen, Armut und Lebensbedingungen. Ergebnisse aus EU-SILC 2005. Wien: Statistik Austria. (2007): http://www.statistik.gv.at/ web_en/ static/einkommen_armut_und_lebensbedingungen_2005_44641_021807.pdf (11.01.2008).

Steinmaurer, Thomas. «Medialer und gesellschaftlicher Wandel. Skizzen zu einem Modell.» Medienentwicklung und gesellschaftlicher Wandel: Beiträge zu einer theoretischen und empirischen Herausforderung. Hrsg. v. Markus Behmer. Wiesbaden: Westdeutscher Verlag, 2003. 103-120.

Vollbrecht, Ralf. «Der sozialökologische Ansatz der Mediensozialisation.» Mediensozialisationstheorien. Neue Modelle und Ansätze in der Diskussion. Hrsg. v. Dagmar Hoffmann u. Lothar Mikos. Wiesbaden: VS Verlag, 2007. 93-108.

Xyländer, Margret; Lange, Andreas; Von der Hagen-Demszky, Alma Mira. «Bildungsprozesse in der frühen Kindheit. Neue Einsichten zur Bedeutung der Familie und ihre Relevanz für den KITA-Alltag.» Familie als Bildungswelt. Theoretische Explorationen und empirische Befunde. Hrsg. v. Andreas Lange u. Margret Xyländer. München: DJI-Verlag, 2008. (im Druck).

Weiss, Ralph. «Auf der Suche nach kommunikativen Milieus. Plädoyer für eine sozialstrukturell interessierte Fragehaltung der Nutzungsforschung.» Zielgruppen, Publikumssegmente, Nutzergruppen. Beiträge aus der Rezeptionsforschung. Hrsg. v. Helmut Scherer u. Hans-Bernd Brosius. München: Reinhard Fischer, 1997. 239-261.

Weiss, Ralph. «'Praktischer Sinn', soziale Identität und Fern-Sehen. Ein Konzept für die Analyse der Einbettung kulturellen Handelns in die Alltagswelt.» Medien und Kommunikationswissenschaft 48. Jg., Heft 1 (2000): 42-62. 
Wegener, Claudia. Stichwort: «Medienforschung in der Erziehungswissenschaft.» Zeitschrift für Erziehungswissenschaft 10.Jg., Heft 4 (2007): 459-477. 